\title{
MINIMAL PATHS AND PROBABILISTIC MODELS FOR ORIGIN-DESTINATION TRAFFIC ESTIMATION IN LIVE CELL IMAGING
}

\author{
T. Pécot ${ }^{1,2}$, C. Kervrann ${ }^{1,2}$, P. Bouthemy ${ }^{1}$ \\ (1) INRIA Rennes Bretagne Atlantique, Campus Universitaire de Beaulieu, F-35042 Rennes \\ (2) INRA, UR341 Mathématiques et informatique appliquées, F-78352 Jouy-en-Josas
}

\begin{abstract}
Green Fluorescent Protein (GFP)-tagging and time-lapse fluorescence microscopy enable to observe molecular dynamics and interactions in live cells. Original image analysis methods are then required to process challenging $2 \mathrm{D}$ or $3 \mathrm{D}$ image sequences. To address the tracking problem of several hundreds of objects, we propose an original framework that provides general information about vesicle transport, that is traffic flows between origin and destination regions detected in the image sequence. Traffic estimation can be accomplished by adapting the advances in Network Tomography commonly used in network communications. In this paper, we address image partition given vesicle stocking areas and multipaths routing for vesicle transport. This approach has been developed for real fluorescence image sequences and Rab proteins.
\end{abstract}

Index Terms- Traffic control (transportation), microscopy, fluorescence, proteins, video, routing, tracking.

\section{INTRODUCTION}

To preserve the structure, cohesion and functions of the org anism, the eukaryotic cell exchanges information between its compartments on physical supports such as intermediate filaments or microtubules. In our study, the transport intermediates corresponding to small spherical vesicles move along microtubules and are propelled by molecular motors. We focus on the traffic between the Golgi apparatus and the endoplasmic reticulum in eukaryotic cells, presumably regulated by two isoforms of the Rab6 GTPase (Rab6A and Rab6A'). Observation of protein dynamics in live cells using GFP-tagging and time-lapse fluorescence video-microscopy can be used to investigate and clarify the role of Rab6A and Rab6A' in retrograde transport [5]. It is worth noting that the GFP-Rab6 proteins are either free (diffusion) in the cytosol, or located at the periphery of the Golgi membrane, or anchored to the vesicle membrane and microtubules (corresponding to traffic).

Image processing methods have been developed to track vesicles over time. The most commonly used tracking concept is the so-called "connexionist" approach [1] which consists in detecting particles independently in each frame in a first step, and then linking the detected objects over time. The related data association task is the most critical step when the number of objects is very high and the trajectories interact. Temporal stochastic filters [2], particle filtering techniques [3] or graph-theory based methods [4] have been then developed to improve temporal matching. In [5], the authors applied a deterministic approach assuming that vesicles are moving along the microtubule network, and thus the number of paths is limited. Kymogram-based modeling is then used for analyzing temporal profiles of different paths. The main limitation of this method is that each path is independently supervised. In [6], the authors propose also to use minimal paths method for estimating the object trajectories without individual object tracking.

In this paper, we propose an alternative and global approach for traffic analysis. The idea is to estimate the number of vesicles going from origin to destination regions. Our estimation method is inspired from the Network Tomography (NT) concept [7] developed for network communications and further applied to video surveillance in [8]. We just need to count the number of "objects/vehicles" in different image regions at each time step. Our contributions are twofolds: i) we extend the usual NT concept described in Section 2 to non-binary routing from geodesic paths given the image sequence; ii) we propose an estimation/optimization framework to derive counting measurements from image intensities (fluorescence) and to solve the traffic flow problem.

\section{TRAFFIC MODEL: NETWORK TOMOGRAPHY}

In time-lapse fluorescence microscopy, GFP-Rab6 proteins involved in traffic correspond to small lighted blobs along the unobserved microtubule network from origin regions (Golgi apparatus) to destination regions ("end-points" located at the periphery of the cell). We decide to adapt the Network Tomography (NT) approach introduced to estimate vehicle traffic flows [9] and re-popularized in computer networks [7] to solve our traffic flow problem.

In this modeling, the network is described as a graph $\mathcal{G}(E, V)$ defined by $n$ vertices and $r$ edges, where $E$ denotes the set of edges (microtubule pieces), and $V$ the set of vertices (vesicle stocking areas or microtubule crossings). Each pair of neighbor vertices is connected by two edges in order to enable traffic in both directions (see Fig. 1 (left)). 


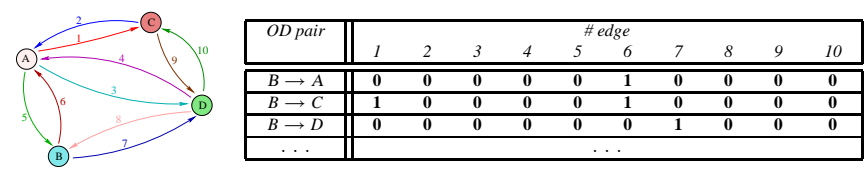

Fig. 1. Lefft: "toy" graph: the vertices are labeled by letters and the edges by numbers; right: Several rows of A corresponding to the "toy" graph.

Then, the vesicles follow a path defined by an origin vertex, a destination vertex, and possible intermediate vertices. The set of paths can be then characterized by the origin and destination vertices, that is the Origin/Destination pairs (OD pairs). Given $n$ vertices in the graph, $c=n(n-1)$ OD pairs are possible. In NT, given the number of objects detected as going from one vertex to a neighbor vertex, the goal is to estimate the proportions of vesicles for each OD pair.

More formally, let $x_{f, t}$ be the number of vesicles on the OD pair \#f at time $t$. The measurements $y_{e, t}$ correspond to the number of vesicles that pass through edge $\# e$ at time $t$. We then assume the following model: $\mathbf{Y}=\mathbf{A X}$, where $\mathbf{Y}=\left\{y_{e, t}\right\}_{e \in\{1, \ldots, r\}, t \in\{1, \ldots, T\}}$ is the set of measurements, $\mathbf{X}=\left\{x_{f, t}\right\}_{f \in\{1, \ldots, c\}, t \in\{1, \ldots, T\}}$ the unknown OD flows, $T$ the number of images and $\mathbf{A}$ denotes the $r \times c$ routing matrix with binary elements $a_{e f}=1$ if edge $e$ belongs to the path for the OD pair \# $f$ (defined as the shortest path in the graph), and 0 otherwise. A cost is then associated to each edge (Euclidean distance between vertices, number of vertices in the path, ...), and the Dijkstra algorithm [10] is applied to the whole graph for computing the shortest path for each OD pair. In Fig. 1 (right), we give several rows of the matrix A corresponding to the graph shown in Fig. 1 (left) when the Euclidean distance between the vertices is considered.

To apply NT in video analysis, we then need to provide a graph given an image sequence, a relevant metric/criterion for computing the routing matrix $\mathbf{A}$ and generate temporal counting measurements in spatial regions to form $\mathbf{Y}$.

\section{GRAPH CONSTRUCTION}

The microtubule network extraction is really hard to perform since we only observe small lighted blobs. Nevertheless, the Maximum Intensity Projection (MIP) map provides useful information about partial trajectories of vesicles and vesicle stocking areas. Accordingly, we extract the origin and destination regions by segmenting the MIP map and labeling the graph vertices.

\subsection{Extraction of origin/destination regions}

The MIP map in the direction of time axis is defined at each point $p$ in the image as: $\operatorname{MIP}(p)=\max _{t \in\{1, \ldots, T\}} I_{t}(p)$, where $I_{t}(p)$ is the intensity observed at point $p$ in the image $I_{t}$. A preliminary pre-processing step [11] is first applied to substract the image background (vesicles free in the cytosol and those anchored at the Golgi membrane). A typical

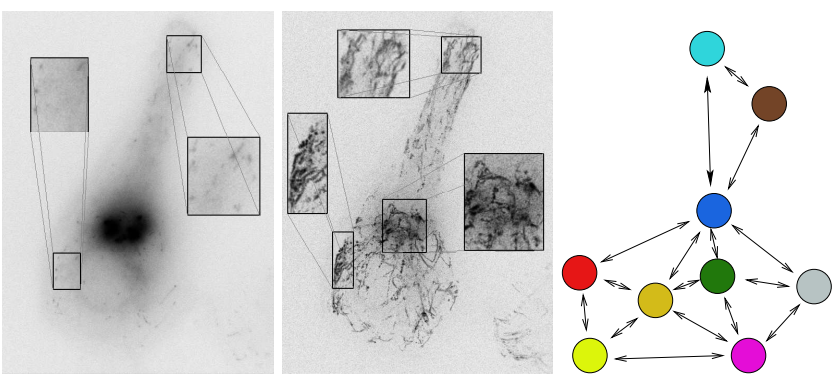

Fig. 2. Image sequence and MIP map. Left) typical image (and zoomed-in views) extracted from a time-lapse microscopy image sequence; middle) MIP map extracted from the sequence corresponding to the image at left (with zoomed-in views of areas of interest). For clarity, the high fluorescence levels are depicted with dark values and a gamma correction is applied for better visualization; right): graph associated to the partition of Fig. 3 a) with colors corresponding to the colors of segmented regions related to the image partition

MIP map is shown in Fig. 2 (middle). The likely origin and destination regions appear as darker and larger regions than moving blobs because vesicles are temporally stocked in these areas. Hence, a segmentation of the MIP map can be used to detect the OD regions in the image. By applying the segmentation method described in [12] to the MIP map of Fig. 2 (middle), meaningful regions are extracted as shown in Fig. 3 a).

In the NT-based approach, the data $\mathbf{Y}$ correspond to the number of vesicles that pass through edges at each time step. Accordingly, we need an image partition composed of adjacent regions for temporal object counting (see [8]).

\subsection{Image partition based on the minimal paths method}

To partition the image, we exploit the minimal paths method [13]. In this setting, the minimal action map computed from a seed point provides for each point in the image a measure proportional to the minimal path between this point and the seed point. Image partition is then achieved by computing a minimal action map for each segmented region. More formally, the minimal action map $\mathcal{U}_{1}$ associated to seed point $p_{1}$ is defined $\forall p \in \Omega$ ( $\Omega$ denotes the MIP map domain) as:

$$
\mathcal{U}_{1}(p)=\min _{\mathcal{A}_{p_{1}, p}}\left\{\int_{\gamma_{p_{1}, p}}\left[v+\mathcal{P}\left(\gamma_{p_{1}, p}(s)\right)\right] d s\right\},
$$

where $\mathcal{A}_{p_{1}, p_{2}}$ is the set of planar curves connecting the points $\left(p_{1}, p_{2}\right) \in \Omega \times \Omega, \gamma_{p_{1}, p_{2}}$ is a curve in $\mathcal{A}_{p_{1}, p_{2}}, v \geq 0$ is a constant and $\mathcal{P}: \Omega \rightarrow \mathbb{R}^{+*}$ gives the intensity at the current point $p$. The partition $\mathcal{G}_{j}$ associated to segmented region of center $p_{j}$ is then derived as: $\mathcal{G}_{j}=\left\{p \in \Omega: \mathcal{U}_{j}(p) \leq \mathcal{U}_{i}(p), \forall i \in\right.$ $\{1, \ldots, N\}, i \neq j\}$. The set of $R$ partitions associated to the segmented regions with centers $\left\{p_{1}, \ldots, p_{R}\right\}$ forms the image partition: $\operatorname{Part}(\Omega)=\bigcup_{j \in\{1, \ldots, R\}} \partial \mathcal{G}_{j}$, where $\partial \mathcal{G}_{j}$ is the boundary of region $\mathcal{G}_{j}$. In practice, we use the fast marching algorithm [13] to compute the minimal action maps. The image partition based on the MIP map of Fig. 2 is shown in Fig. 3 a). 


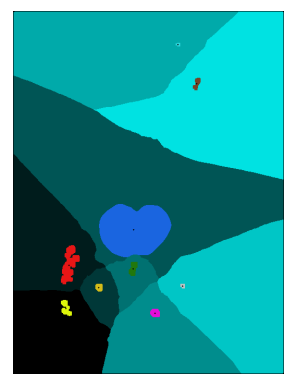

a)

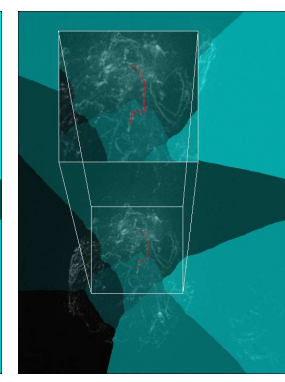

b)

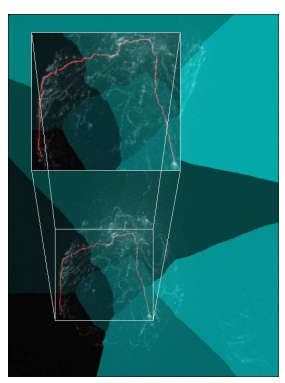

c)
Fig. 3. a) image partition based on the minimal paths method applied to the MIP map shown in Fig. 2 (right) given the seed points (labeled by black crosses) defined as gravity centers of the segmented regions (appearing as colored areas); b-c) minimal paths computed for connecting region pairs (with zoomed-in views). The MIP map appears as a bright region in the background and the minimal paths are depicted in red. In a), b) and c), the regions $\mathcal{G}_{j}$ (image partition) are depicted with light-to-dark green variations.

Given a partition of the MIP map, we define the vertices (regions) and edges (region boundaries) to form a graph (see Fig. 2 (right)). In addition, we also need to define a cost for each edge to derive the routing matrix $\mathbf{A}$.

\subsection{Costs for edges}

The minimal paths between the gravity centers of neighboring regions can be used to define the edge costs. Let $\mathrm{MP}_{1,2}$ be the minimal path between the gravity centers $p_{1}$ and $p_{2}$ :

$$
\mathrm{MP}_{1,2}=\min _{\mathcal{A}_{p_{1}, p_{2}}}\left\{\int_{\gamma_{p_{1}, p_{2}}}\left[v+\mathcal{P}\left(\gamma_{p_{1}, p_{2}}(s)\right)\right] d s\right\} .
$$

To get $\mathrm{MP}_{1,2}$, the minimal action map $\mathcal{U}_{1}$ (resp. $\mathcal{U}_{2}$ ) is first computed. A gradient descent is then applied from $p_{2}$ (resp. $p_{1}$ ). We define the edge cost between the regions \#1 and \#2 as the integral of intensity of the MIP map along the minimal path. Two typical examples of minimal paths used to define path costs are depicted in Fig. 3 b) and c). In the first example, a relatively short path is computed while in the second example, the path is much longer than the Euclidean distance between the two region centers. This demonstrates that a few vesicles are moving directly through the common boundary $\# \partial_{1,2}$. These costs will be used to derive an original form for the routing matrix $\mathbf{A}$.

\section{TRAFFIC ESTIMATION}

In our study, it is established that the level of fluorescence is proportional to the number of vesicles at each pixel. Our idea to infer the number of vesicles passing through each edge (i.e. Y) amounts to computing the difference of intensity variation at two consecutive time steps in each neighboring region.

\section{1. $Y$ computation}

We consider the fluorescence exchanges at vertex $A$ in the graph shown in Fig. 1 (left). Let $z_{v, t}$ be the total amount of

\begin{tabular}{|c||cccccccccc|}
\hline \multicolumn{1}{|c||}{ OD pair } & \multicolumn{10}{|c|}{ \# edge } \\
& 1 & 2 & 3 & 4 & 5 & 6 & 7 & 8 & 9 & 10 \\
\hline \hline$A \rightarrow B$ & $\frac{m_{3}}{M}$ & 0 & $\frac{m_{2}}{M}$ & 0 & $\frac{m_{1}}{M}$ & 0 & 0 & $\frac{m_{2}+m_{3}}{M}$ & $\frac{m_{3}}{M}$ & 0 \\
\hline
\end{tabular}

Table 1. Non-binary routing matrix for the OD pair $A \rightarrow B$ corresponding to the graph shown in Fig. 1 (left). We associate the path $A \rightarrow B$ to the probability $m_{1}$, $A \rightarrow D \rightarrow B$ to $m_{2}$, and $A \rightarrow C \rightarrow D \rightarrow B$ to $m_{4}$, and $M=m_{1}+m_{2}+m 3$.

fluorescence in the complete region corresponding to vertex $v$ at time $t$, and let $y_{e, t}$ be the fluorescence intensity to be determined on edge $e$ at time $t$. Then we observe: $z_{A, t+1}-$ $z_{A, t}=y_{2, t+1}-y_{1, t+1}+y_{4, t+1}-y_{3, t+1}+y_{6, t+1}-y_{5, t+1}$.

This equation can be extended to all vertices: let $\Delta \mathbf{Z}$ be the $R \times T$ matrix denoting the difference of fluorescence intensity in each region between two consecutive time steps. Let $\mathbf{Y}$ be the $r \times T$ matrix representing the level of fluorescence that fluctuates on edges at each time step. We define $\mathbf{M}$ as the so-called "neighborhood $n \times r$ matrix" composed of ternary elements $m \in\{-1,0,1\}$ that expresses neighborhood relationships. Then, we have $\Delta \mathbf{Z}=\mathbf{M Y}$, and the problem is under-constrained $(r>n)$. We assume that all the components of $\mathbf{Y}$ are positive since $\mathbf{Y}$ represents counts. Finally, the measurements are obtained by solving the following optimization problem:

$$
\widehat{\mathbf{Y}}=\min _{\mathbf{Y}}\|\Delta \mathbf{Z}-\mathbf{M Y}\|^{2} \text { subject to } \mathbf{Y} \geq \mathbf{0} \text {. }
$$

\subsection{Probabilistic routing matrix}

In our study, it is also desired that the vesicles can follow different paths for going from an origin to a destination region. For each OD pair, we compute all the paths linking the origin to the destination thanks to a depth-first search in the graph. To each path, we associate a cost defined as the sum of edge costs. Unlike to the usual NT [7], we consider a probabilistic routing matrix and propose the following prior probabilities $m_{w} \propto \exp \left(-\frac{c_{w}}{\sigma}\right)$, where $c_{w}$ denotes the cost of path $w$, and $\sigma$ is a constant. In practice, $\sigma$ is different for each OD pair and is chosen to be the smallest path cost for the current OD pair to encourage the shortest paths. The probabilistic routing matrix is then derived as follows. Let $\left\{w_{e}\right\}_{i}, i \in\{1, \ldots, Q\}$, be the set of paths that use the edge $e$ and let $m_{\left(w_{e}\right)_{i}}$ be the corresponding probability. Hence, each element $a_{e, f}$ of the non-binary routing matrix $\mathbf{A}$ is defined as $a_{e, f}=\frac{\sum_{i=1}^{Q} m_{\left(w_{e}\right)_{i}}}{\sum_{e^{\prime}=1}^{r} \sum_{i=1}^{Q} m_{\left(w_{e^{\prime}}\right)_{i}}}$.

For illustration, the row of the non-binary routing matrix for the OD pair $A \rightarrow B$ in the graph shown in Fig. 1 (left) is given in Tab.1.

\subsection{NT optimization}

In our study, we are only interested in the proportions of vesicles on each OD pair for the whole image sequence. So we 
propose instead to solve the following optimization problem:

$$
\min _{\overline{\mathbf{x}}}\|\overline{\mathbf{y}}-\mathbf{A} \overline{\mathbf{x}}\|^{2} \quad \text { s.t. } \bar{x}_{f} \geq 0, f \in\{1, \ldots, c\},
$$

where $\overline{\mathbf{x}}=\left(\bar{x}_{1}, \ldots, \bar{x}_{c}\right)^{T}$ contains the positive proportions of vesicles for each OD pair and $\overline{\mathbf{y}}=\left(\bar{y}_{1}, \ldots, \bar{y}_{r}\right)^{T}$ defined as $\bar{y}_{e}=\frac{1}{T} \sum_{t=1}^{T} y_{e, t}, \quad \forall e \in\{1, \ldots, r\}$ correspond to temporal averages. As the traffic is observed only on a few of OD pairs, we add a parsimony constraint and solve the following problem:

$$
\min _{\overline{\mathbf{x}}}\|\overline{\mathbf{y}}-\mathbf{A} \overline{\mathbf{x}}\|^{2}+\lambda\|\overline{\mathbf{x}}\|_{\mathbf{0}} \quad \text { s.t. } \bar{x}_{f} \geq 0, f \in\{1, \ldots, c\},
$$

where $\|\overline{\mathbf{x}}\|_{0}=\#\left\{\bar{x}_{i} \neq 0\right\}, i \in\{1, \ldots, c\}$. An alternative to express the parsimony constraint is to introduce hard constraints about the origin or destination vertices if known. For example, assume that vertex $r$ is the single origin region. Let $\mathcal{R}$ be all the OD pairs that have $r$ for origin and $\mathcal{O}$ the set of all OD pairs. The routing matrix $\mathbf{A}$ is then updated as follows: $\mathbf{A}(e, \mathcal{O} \backslash \mathcal{R})=0, \forall e \in\{1, \ldots, r\}$. The same restriction can be applied to impose additional origin or destination vertices. Once the routing matrix is updated, the problem (1) is solved by standard non-negative mean square minimization.

\section{EXPERIMENTAL RESULTS}

The NT concept was successfully experimented on Rab6 traffic simulations in [14]. We propose four experiments corresponding to the sequence composed of 121 images (2-bytes) using time-lapse fluorescence (wide-field) microscopy (resolution: $160 \mathrm{~nm} \times 160 \mathrm{~nm}$, frame rate: 2 images/sec.), and shown in Fig. 2 (left). In a preliminary step, the background was removed using [11]. The traffic estimation results are reported in Fig. 4. In this figure, the black region corresponds to a masked area, consequently the fluorescence variations in this region are not taken into account.

In Fig. 4 a) and b), we compare the estimated OD flows between a single path routing matrix and a probabilistic routing matrix. According to the expert-biologists, the vesicles mostly move from the Golgi Apparatus (blue region) to "endpoints" located at the periphery of the cell. With a single path routing, a lot of significant OD pairs are estimated in any directions with no preference. This result is not satisfying and is not consistent with the prior knowledge given by the experts. By considering a probabilistic routing matrix, a limited number of significant OD pairs are estimated, mainly oriented from the image center to the periphery.

For the experiments shown in Fig. 4 c) and d), the Golgi apparatus region (in blue) is compelled to be an origin region. In these experiments, two different partitions/segmentations are used. In both experiments, three principal directions for the traffic are extracted with roughly similar estimated traffic flows: from the Golgi apparatus to respectively the top (22\%$24 \%)$, left bottom $(41 \%-30 \%)$ and right bottom $(37 \%-46 \%)$ of the figure. This tends to show that traffic flows are hierarchically organized and "motorways" can be identified.

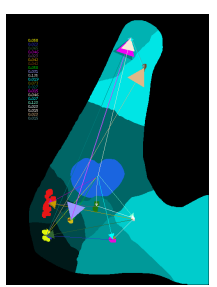

a)

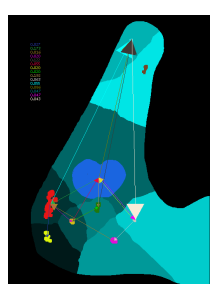

b)

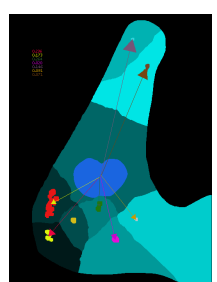

c)

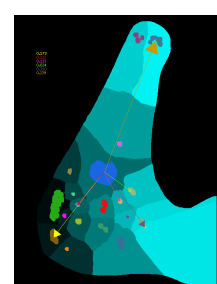

d)
Fig. 4. Results obtained by applying the NT-based approach on the sequence shown in Fig. 2 (left). The colored areas correspond to the segmented regions and the partition is labeled with light-to-dark green variations. The arrows represent the estimated OD pairs, and the corresponding colored numbers at the left top represent traffic proportions.

\section{CONCLUSION}

In this paper, we have proposed a general framework for traffic flow estimation without individual tracking of moving objects. First, an image partition is performed from a crude segmentation of the OD regions using the minimal paths method. Then, an adjacency graph with edge costs proportional to the path lengths is computed. Finally, we solve the traffic flow problem by considering a probabilistic routing matrix and temporal counting measurements to extract meaningful paths corresponding to "motorways" for vesicle trafficking in molecular imaging. More intensive experiments have been conducted but cannot be reported in this paper.

\section{REFERENCES}

[1] I.F. Sbalzarini and P. Koumoutsakos, "Feature point tracking and trajectory analysis for video imaging in cell biology," J. Struct. Biol., vol. 151, pp. 182-195, 2005.

[2] A. Genovesio, T. Liedl, V. Emiliani, W.J. Parak, M. Coppey-Moisan, and J.-C. Olivo-Marin, "Multiple particle tracking in 3D+t microscopy: Method and application to the tracking of endocytosed quantum dots," IEEE Trans. on IP, vol. 15, no. 5, pp. 1062-1070, 2006.

[3] I. Smal, K. Draegestein, N. Galjart, W.J. Niessen, and E.H.W. Meijering, "Raoblackwellized marginal particle filtering for multiple object tracking in molecular bioimaging," in Proc. of IPMI'07, 2007, pp. 110-121.

[4] D. Thomann, J. Dorn, P.K. Sorger, and G. Danuser, "Automatic fluorescent tag localization ii: improvement in super-resolution by relative tracking," J. Microscopy, vol. 211, no. 3, pp. 230-248, 2003.

[5] V. Racine, M. Saschse, J. Salamero, V. Fraisier, A. Trubuil, and J.B. Sibarita, "Visualization and quantification of vesicle trafficking on a $3 \mathrm{~d}$ cytoskeleton network in living cells," J. Microscopy, pp. 214-228, Mar. 2007.

[6] S. Bonneau, M. Dahan, and L.D. Cohen, "Tracking single quantum dots in live cells with minimal paths," in Proc. of CVPR'05, 2005, vol. 3, pp. 141-141.

[7] Y. Vardi, "Network tomography: Estimation source-destination traffic intensities from link data," J. Amer. Statist. Ass., vol. 91, no. 433, pp. 365-377, Mar. 1996.

[8] J. E. Boyd, J. Meloche, and Y. Vardi, "Statistical tracking in video traffic surveillance," in Proc. of ICCV'99, 1999, vol. 1, pp. 163-168.

[9] E. Cascetta and S. Nguyen, "A unified framework for estimating or updating origin/destination matrices from traffic counts," Transportation Research, vol. 22, no. 6, pp. 437-455, 1988.

[10] E.W. Dijkstra, "A note on two problems in connexion with graphs," Numerische Mathematik, vol. 1, no. 1, pp. 269-271, 1959.

[11] J. Boulanger, C. Kervrann, and P. Bouthemy, "Estimation of dynamic background for fluorescence video-microscopy," in Proc. of ICIP'06, Atlanta, 2006.

[12] C. Kervrann and A. Trubuil, "Optimal level curves and global minimizers of cost functionals in image segmentation," J. Math. Imag. Vis., vol. 17, pp. 153-174, 2002.

[13] L.D. Cohen, "Minimal paths and fast marching methods for image analysis," Mathematical Models in Computer Vision: the Handbook, to appear.

[14] T. Pécot, J. Boulanger, C. Kervrann, and P. Bouthemy, "Network tomography for trafficking simulation and analysis in fluorescence microscopy imaging," in Proc. of IEEE ISBI'07, Arlington, 2007, pp. 268-271. 